\title{
Autologous Adipose-Derived Mesenchymal Stem Cells Embedded in Platelet-Rich Fibrin in Diabetic Foot Ulcers
}

\author{
Charbel Khalil1,2,3*, Diana Chaker,4,5, Rawad Salameh ${ }^{1}$, Yvonne-Elise Germanos ${ }^{6}$, \\ Elie El Kayem7 , Fady Nader6, Jessica Habbouche2,8, Rodrigue Chemaly ${ }^{2,6,7}$, Albert Azar' \\ Ahmad Ibrahim 1,2,6,9
}

\author{
${ }^{1}$ Reviva Stem Cell Platform for Research and Applications Center, Bsalim, Lebanon \\ ${ }^{2}$ Middle East Institute of Health University Hospital, Bsalim, Lebanon \\ ${ }^{3}$ Faculty of Pharmacy, Saint Joseph University, Beirut, Lebanon \\ ${ }^{4}$ National Institute of Health and Medical Research, INSERM, Paris Sud, France \\ ${ }^{5}$ Faculty of Pharmacy, Therapeutic Innovation from Fundamentals to Applications, Paris Saclay University, \\ Chätenay Malabry, France \\ ${ }^{6}$ Faculty of Medicine, Lebanese University, Beirut, Lebanon \\ ${ }^{7}$ School of Medicine, Lebanese American University, Beirut, Lebanon \\ ${ }^{8}$ Michigan Medicine Speciality Pharmacy, Ann Arbor, MI, USA \\ ${ }^{9}$ Cancer Center, Middle East Institute of Health University Hospital, Bsalim, Lebanon \\ Email: *charbelk3@hotmail.com
}

How to cite this paper: Khalil, C., Chaker, D., Salameh, R., Germanos, Y.-E., El Kayem, E., Nader, F., Habbouche, J., Chemaly, R., Azar, A. and Ibrahim, A. (2021) Autologous Adipose-Derived Mesenchymal Stem Cells Embedded in Platelet-Rich Fibrin in Diabetic Foot Ulcers. Open Journal of Regenerative Medicine, 10, 19-30. https://doi.org/10.4236/ojrm.2021.102002

Received: April 14, 2021

Accepted: June 27, 2021

Published: June 30, 2021

Copyright $\odot 2021$ by author(s) and Scientific Research Publishing Inc. This work is licensed under the Creative Commons Attribution International License (CC BY 4.0).

http://creativecommons.org/licenses/by/4.0/ (c) (i) Open Access

\begin{abstract}
Context and Aim: Mesenchymal stem cells (MSCs) and platelet-rich fibrin (PRF) have emerged as ideal candidates for advanced therapies of various therapeutically-challenging diseases; however, their regenerative potential in diabetic foot ulcers (DFU) has not been well determined. In this study, we reviewed our clinical experience in mitigating chronic ulcer complications of diabetic foot through a conventional treatment of autologous adipose-derived MSCs embedded in PRF with pure PRF injections. Materials and Methods: The present study was carried out in 10 patients with an open DFU wound selected over a period of 1 year starting from April 2019. Patients were either injected with PRF alone (Group A) or injected with MSCs derived from adipose tissue (ADMSC) embedded in (PRF (Group B). Results: Patients in Group B had a better healing index when compared to Group A. Conclusion: Use of ADMSC embedded in PRF showed promising results to treat DFU.
\end{abstract}

\section{Keywords}

Diabetic Foot Ulcer, Diabetes, Mesenchymal Stem Cells, Adipose Tissue, Platelet Rich Fibrin 


\section{Introduction}

Diabetic foot infections have been an increasingly common problem globally due to the sustained rise in new incidences of diabetes and the increasing body weight of diabetic patients [1] [2]. They usually arise either as a skin ulceration caused by a sensory or motor peripheral neuropathy or as a trauma-induced wound [1]. Diabetic foot ulcer (DFU) complications are the main reason for diabetes-related hospitalizations and lower extremity amputations [1]. In the United States, DFUs constitute two-thirds of all non-traumatic amputations [3]; while in Lebanon, $80 \%$ of patients with DFUs undergo a lower limb amputation [4]. It is considered a major public health issue because it negatively impacts patients physically and emotionally and involves a large proportion of costs to the medical system and society [1] [3].

Patients with open wounds require particular local-wound care [5]. Following tissue injury, cellular and chemotactic factors promote the migration of fibroblasts, macrophages, keratinocytes, and platelets to the site of injury accompanied with endothelial cell recruitment that is essential for angiogenesis [6] [7] [8]. The process of wound healing is characterized to be a linear pathway that starts with the achievement of hemostasis, followed consecutively by inflammation, epithelialization, and fibroplasia to complete the healing process [9] [10]. In order to manage and maintain wound healing, the cells release growth factors (GF) and cytokines, such as VEGF, FGF2, TGF- $\beta$, etc. [11].

Although many topical agents and wound maintenance interventions are meant to improve the wound healing environment, such as debridement, hyperbaric oxygen therapy, ultrasound and electromagnetic energy [9] [10], negative pressure wound therapy [11], extracellular matrix products and cellular-based tissue technologies [12], etc. unfortunately data is lacking to support any definitive protocols for DFU healing.

In the past decade, various studies have demonstrated that the therapeutic use of platelet-rich plasma (PRP) was successful as a basic sealing and hemostatic therapeutic tool in plastic surgery especially in oral, hand, and aesthetic surgery [12] [13] [14]. However, many limitations in PRP therapy, such as the association of anti-coagulants, have the benefits and clinical outcomes of PRP use in the treatment of chronic ulcers, resulting in the inhibition of the wound healing process [15] [16]. Thus, platelet-rich fibrin (PRF) was developed as an easy to prepare, nontoxic, and biocompatible platelet-based therapeutic alternative [17] [18]. Also known as "Glue Therapy", PRF used as a cell scaffold was proposed as an effective approach to promote and repair wound healing [6]. PRF is a platelet concentrate collected on a single fibrin membrane that contains all the constituents favorable for healing. The scientific rationale behind the use of platelet preparations lies in the fact that platelets serve as a reservoir for numerous growth factors that are known to play a crucial role in hard-tissue and soft-tissue healing processes [7]. Intact platelets contained within a fibrin matrix release a relatively constant concentration of growth factors over a period of 7 days, stimulating human osteoblastic proliferation. An effect on neoangiogenesis also has been 
shown through histology. In a membrane form, platelet can be used as a fibrin bandage that serves as a matrix to accelerate the healing of wound edges [8]. Platelets are considered to play an important role in the healing process of wounds, as they release GFs upon activation following their pulverization on the wound bed within the first hour [19]. These GFs contribute to the repair of injured tissues through promoting angiogenesis, synthesis of extracellular matrix components like collagen, laminin, and integrin, and interestingly via inducing a re-epithelization of the injured site [12] [20].

Recently, mesenchymal stem cells (MSC) have been used in many clinical trials and applications giving rise to various differentiated cell lineages [13]. MSCs can be collected from bone marrow, umbilical cords [14], and from adipose tissue (ADMSC), which is a less invasive and painful method than harvesting human bone marrow [21]. MSC-based therapies are also promising alternatives to treat tissue loss diseases through their high proliferation potential and cytokine and growth factor secretions known to be essential for organ homeostasis and tissue repair [17]. Like platelets, MSCs also promote healing in DFUs by secreting GFs, which play a role in reducing inflammation and promoting angiogenesis within the wound [21].

In this study, we evaluated the efficacy of ADMSCs embedded in autologous PRF on the chronic DFU healing process compared to autologous PRF treatment alone. We propose our protocol as a potentially new cost-effective therapeutic approach with better and faster outcomes than traditional chronic wound healing treatments.

\section{Material and Methods}

\subsection{Study Design and Patient Selection}

This is a retrospective, experimental study. Ten patients with chronic DFU were divided into two groups (each $n=5$ ): Group A (pure PRF) and Group B (ADMSC + PRF). The administration of treatment took place on an outpatient basis without the need for hospital admission. Between two and three sessions were performed per patient (once a week). All participants were aged between 53 and 93 years old (median age \pm 71 years old) and were refractory to the usual conservative DFU treatments, such as enzymatic and antibiotic debriding substances and various hydrocolloid dressings. There were no exclusion criteria; all patients with diabetic foot ulcers were included even those with infected wounds.

All procedures conducted during the study were carried out in compliance with institutional ethical standards. Reviva Regenerative Medicine Center and the Middle East Institute of Health-University Hospital Ethics Review Boards approved the retrieval of all MSC and PRF collections (Approval Reference Number CTU-009-15) and all patients were asked to read, approve, and sign an informed consent form prior to any participation.

To evaluate ulcer progression and examine the regenerative power of the PRF, we used the "Zaragoza 1-4" scale: Table 1. 
Table 1. Zaragoza scale.

\begin{tabular}{ccc}
\hline Score & Clinical Observation & Outcome \\
\hline $\mathbf{0}$ & No improvement & Very Poor \\
$\mathbf{1}$ & Granulation only on peripheral edges & Poor \\
$\mathbf{2}$ & Deep-seated granulation only & Fair \\
$\mathbf{3}$ & Adequate deep-seated and surface granulation & Good \\
$\mathbf{4}$ & Complete healing & Excellent \\
\hline
\end{tabular}

\subsection{Preparation of Autologous PRF}

We used the Vivostat ${ }^{\circledR}$ PRF system, an automated system for efficient preparation and application of autologous PRF in wound treatment. Blood $(120 \mathrm{~mL})$ was drawn into a preparation unit, anticoagulants were added, and the whole preparation unit was double-centrifuged to obtain $5 \mathrm{~mL}$ of plasma rich in growth factors and autologous fibrin (concentration $20 \mathrm{mg} / \mathrm{mL}$ ). This process took approximately 23 minutes $(\mathrm{min})$. Samples were then taken for culture of the wounds and the ulcers were disinfected using a normal saline and chlorhexidine $2 \%$ wash. Photographs were taken to allow monitoring of the course of treatment. (Note: No thrombin or bovine components were added to the Vivostat ${ }^{\circledR}$ PRF at any time.)

\subsection{Mesenchymal Stem Cell Collection}

Patients underwent standard liposuction under good medical practice conditions. Approximately $250 \mathrm{~mL}$ of fat mixed with local anesthetics was obtained. To isolate the stromal vascular fraction (SVF), lipoaspirates were washed intensely with phosphate-buffered saline (PBS) containing $5 \%$ of penicillin, streptomycin and amphotericin B (PSA). Next, the lipoaspirates were digested with an equal volume of $0.075 \%$ collagenase type I (Sigma-Aldrich) for $30-60 \mathrm{~min}$ at $37^{\circ} \mathrm{C}$ with gentle agitation. The activity of the collagenase was neutralized with Dulbecco's Modified Eagle Medium (DMEM) containing 10\% fetal bovine serum (FBS; Invitrogen; Thermo Fisher Scientific, Inc., Waltham, MA, USA). To obtain the high-density SVF pellet, the digested lipoaspirate was centrifuged at $1200 \mathrm{~g}$ for $10 \mathrm{~min}$. The pellet was then resuspended in DMEM containing 10\% FBS and filtered through a $100 \mu \mathrm{m}$ nylon cell strainer (Falcon). The filtered cells were centrifuged at $1200 \mathrm{~g}$ for $10 \mathrm{~min}$. The resuspended SVF cells were plated at a density of $1 \times 10^{6} / \mathrm{cm}^{2}$ into T75 or T175 culture flasks. Non-adherent cells were removed $12-18$ hours after initial plating by intensely washing the plates. The resulting fibroblastoid adherent cells, termed AT-derived fibroblastoid adherent cells, were cultivated at $37^{\circ} \mathrm{C}$ at a humidified atmosphere containing $5 \% \mathrm{CO}_{2}$. The expansion medium consisted of Minimum Essential Medium Eagle - Alpha Modification (Alpha MEM) + 10\% fetal bovine serum (FBS; Invitrogen; Thermo Fisher Scientific, Inc., Waltham, MA, USA) and 5\% penicillin-streptomycinamphotericin B solution (PSA: Hyclone; GE healthcare, Logan, UT, USA). ADMSCs were maintained in Alpha-MEM + 10\% FBS and 5\% PSA until they 
reached $70 \%$ to $90 \%$ confluency. Cells were harvested at subconfluence using Trypsin (Sigma-Aldrich).

\subsection{Platelet Concentrate Functionality Evaluation}

TGF- $\beta 1$, PDGF-AB, PDGF-BB, VEGF and FGF- 2 were measured by ELISA. The supernatant and the antibody (monoclonal biotinyle) form a complex after the interaction of biotin and streptavidin during incubation for $60 \mathrm{~min}$. The excess was eliminated by washing and then an enzyme-antibody complex was added. The complex antibody-enzyme formed the final sandwich complex. After incubation, the excess was washed again. Afterwards, sulfuric acid was added to stop the reaction and the solution's color transformed from blue to yellow. The color intensity was directly correlated to the concentration of samples. The absorbance was read at $450 \mathrm{~nm}$.

\subsection{Flow Cytometry Analysis for ADMSC Characterization and Immunophenotyping}

For MSC surface marker immunophenotyping, cells were stained with the following conjugated antibodies: anti-CD45-vioblue, anti-CD34-PE, HLADr-vioblue, anti-CD73-PE, anti-CD90-FITC, anti-CD105-vioblue, CD24-PE, CD44-FITC, CD133-APC, CD14-PE and relevant isotypes (Miltenyi-Biotec). We acquired at least 20,000 events as test samples.

\subsection{Mesenchymal Stem Cells Embedded in PRF}

The platelet-rich fibrin obtained after preparation were mixed with $10 \times 10^{6}$ cells of ADMSC.

\subsection{Injection Procedure}

The Vivostat ${ }^{\circledR}$ PRF syringe was loaded in an Applicator Unit and applied on the wound with the Spraypen by a minimum distance of $5 \mathrm{~cm}$ from the problem area until it was well covered. The final dressing was with Bactigras ${ }^{\circledR}$ covered with a suitable antiperspirant. The dressing was not removed until the following session.

\section{Statistical Analysis}

Statistical significance was determined based on a paired $T$-test and one-way ANOVA test. $P$ values below 0.05 were considered as statistically significant. Chi-square test was used to assess wound healing scores in the two groups.

\section{Results}

The present study was carried out with 10 patients selected from the outpatient department over a period of 1 year starting from April 2019 with DFUs. The patients were divided into Group A treated with PRF alone $(n=5)$ and Group B treated with PRF embedded in ADMSC $(n=5)$ (Table 2). 
Table 2. Description of clinical cases.

\begin{tabular}{|c|c|c|c|c|c|c|c|}
\hline Case & Age & Sex & $\begin{array}{l}\text { Risk } \\
\text { Factor }\end{array}$ & Etiology & $\begin{array}{l}\text { Injection } \\
\text { Location }\end{array}$ & $\begin{array}{l}\text { Number of } \\
\text { Sessions }\end{array}$ & $\begin{array}{l}\text { Type of } \\
\text { Injection }\end{array}$ \\
\hline 1 & 93 & M & $\begin{array}{l}\text { Hematological Disease (Essential } \\
\text { Thrombocythemia) + Diabetes }\end{array}$ & Pressure & Maleola & 3 & PRF \\
\hline 2 & 87 & M & Venous Insufficiency + Diabetes & Post-Surgical & Knee Rupture & 2 & PRF \\
\hline 3 & 85 & M & Renal Insufficiency + Diabetes & Burn & Lower limb & 3 & PRF \\
\hline 4 & 82 & M & Paraplegia + Diabetes & Pressure & Sacrum & 2 & PRF \\
\hline 5 & 75 & $\mathrm{~F}$ & Paraplegia + Diabetes & Pressure & Sacrum & 2 & PRF \\
\hline 6 & 55 & $\mathrm{~F}$ & Venous Insufficiency + Diabetes & Post-Surgical & Lower limb & 2 & $\mathrm{PRF}+\mathrm{ADMSC}$ \\
\hline 7 & 62 & $\mathrm{~F}$ & Colon Cancer + Diabetes & Post-Surgical & Sacrum & 2 & $\mathrm{PRF}+\mathrm{ADMSC}$ \\
\hline 8 & 59 & M & Venous Insufficiency + Diabetes & Vascular & Sacrum & 2 & $\mathrm{PRF}+\mathrm{ADMSC}$ \\
\hline 9 & 60 & M & Venous Insufficiency + Diabetes & Vascular & Lower limb & 2 & PRF + ADMSC \\
\hline 10 & 53 & M & Venous Insufficiency + Diabetes & Post-Surgical & Sacrum & 3 & $\mathrm{PRF}+\mathrm{ADMSC}$ \\
\hline
\end{tabular}

A significantly higher proportion of individuals in the ADMSC $+\mathrm{PRF}$ group had a better healing index when compared to the pure PRF group $(P<0.001)$ (Table 3).

Hence, in the present study, DFU grafted with ADMSC + PRF showed a statistically significant difference in wound healing; however, clinical significance was also observed in the experimental group with pure PRF grafting.

\subsection{Platelet Concentrate in PRF}

The mean concentration of TGF- $\beta 1$, PDGF-AB, PDGF-BB, VEGF and FGF-2 secreted by the PRF were $12,500 \mathrm{pg} / \mathrm{mg}, 3200 \mathrm{pg} / \mathrm{mg}, 400 \mathrm{pg} / \mathrm{mg}, 28 \mathrm{pg} / \mathrm{mg}$ and $4.2 \mathrm{pg} / \mathrm{mg}$ respectively compared to patient serum used as a control (CTR) (Figure 1).

\subsection{PRF Induces MSC Proliferation}

All patients showed multiple skin lesions with a median initial size of at least 5 $\mathrm{cm}^{2}$, involving the dermis (Grade 1) and subcutaneous (Grade 2) layers. Microbiological assessment was positive in one case, in which antibiotics were added on to the PRF injection (Table 4). A complete response was observed in 4 patients after a mean of 3 sessions. The other 4 patient achieved a partial response. Two patients stopped the gel treatment too early due to low patient compliance or death (Figure 2). Pain disappeared in all cases after the 2nd gel application, while granulation tissue was observed after the first application in 3 patients with Grade 2 lesions (Figure 3, Figure 4) injected with PRF alone and 4 patients injected with PRF embedded in ADMSC (Table 4). No side effects were documented. 
Table 3. Intergroup comparison of wound healing score after 6 weeks.

\begin{tabular}{ccc}
\cline { 2 - 3 } Wound Healing Index & $\begin{array}{c}\text { Group A (n = 5) } \\
P R F \text { Alone }\end{array}$ & $\begin{array}{c}\text { Group B (n= 5) } \\
A D M S C+P R F\end{array}$ \\
\cline { 2 - 3 } & Very poor (Score 1) & 0 \\
Poor (Score 2) & 1 & 0 \\
Good (Score 3) & 0 & 1 \\
Very Good (Score 4) & 4 & 0 \\
Excellent (Score 5) & 0 & 4 \\
\hline
\end{tabular}

$\mathrm{P}$ value highly significant $(\mathrm{P}<0.001)$ and obtained by Chi-square test.

Table 4. Influence of the infection variable in each patient case.

\begin{tabular}{ccccc}
\hline Case & Infection PRE Injection & Infection POST Injection & Sessions & Score \\
\hline 1 & Yes & No & 3 & 4 \\
2 & No & No & 2 & 4 \\
3 & No & No & 3 & 3 \\
4 & No & No & 2 & 3 \\
5 & No & No & 2 & 3 \\
6 & No & No & 2 & 3 \\
7 & No & No & 2 & 3 \\
8 & No & No & 2 & 3 \\
9 & No & No & 2 & 3 \\
10 & No & No & 3 & 2 \\
\hline
\end{tabular}

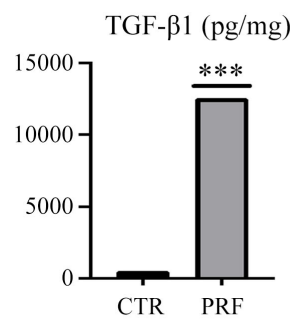

(a)

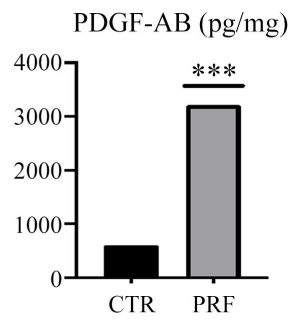

(b)

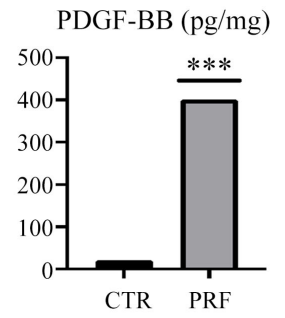

(c)

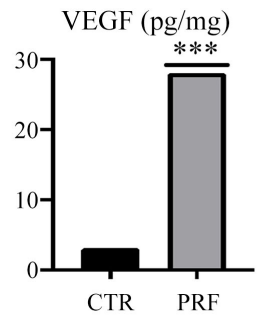

(d)

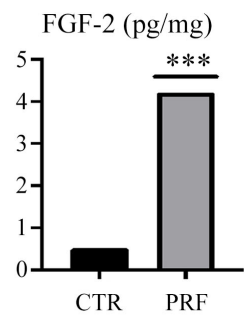

(e)

Figure 1. Concentrations (mean \pm SEM) of the five selected growth factors in CTR (serum) and Vivostat ${ }^{\circledR}$ PRF nortmalized to total soluble protein. ${ }^{* * *} p<0.001$ versus serum (paired $t$-test).

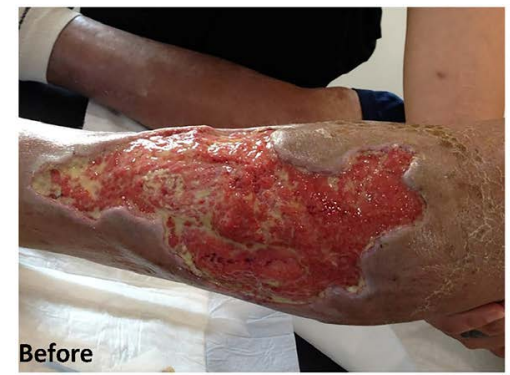

(a)

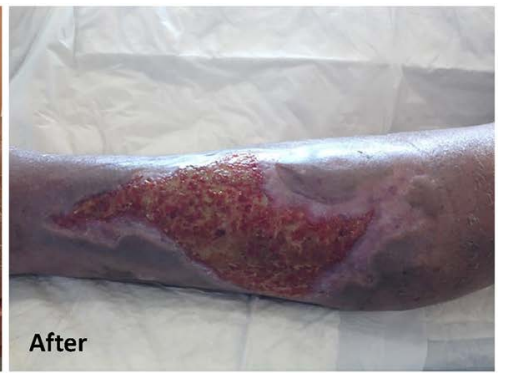

(b)

Figure 2. Patient with diabetic fot ulcer before treatment initation (a) and 3 months after PRF injection (b). 


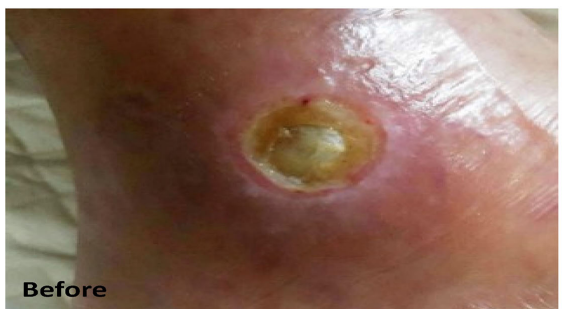

(a)

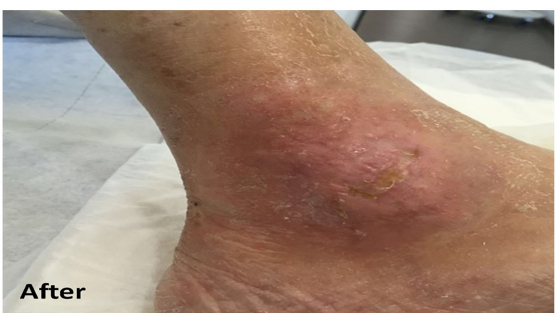

(b)

Figure 3. Patient with diabetic foot ulcer before treatment initiation (a) and 4 months after PRF injection (b).

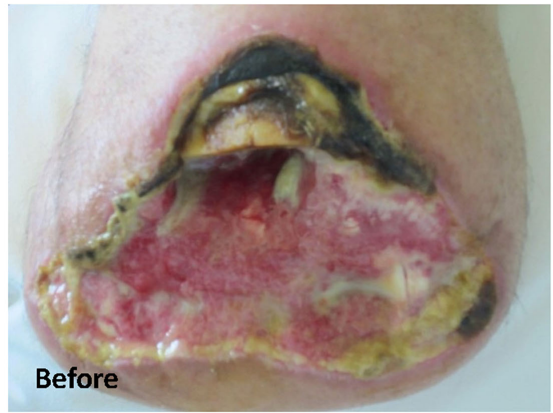

(a)

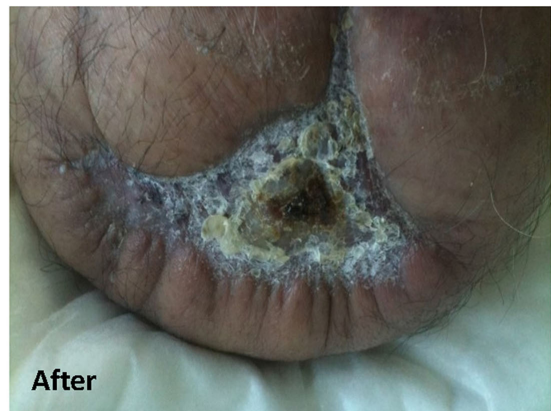

(b)

Figure 4. Patient with a leg amputation before treatment initation (a) and 8 weeks post ADMSC + PRF treatment (b).

\section{Discussion}

Several research studies have discussed DFU healing processes; however, most of these studies are focused on evaluating regular dressings, such as debridement, antibacterial agents, tulle dressing, polyurethane films, foam, etc. Over recent years, improvement in tissue regeneration therapy in Lebanon has involved the use of PRP in therapeutic processes as a valid application in reconstructive and aesthetic medicine. The therapeutic tool evolution of switching from PRP to PRF cell product has shown the robust potential of PRF as a hemostatic, anti-inflammatory, and analgesic substance [18] [22]. MSC-based treatment also increases wound tensile strength, reduces scarring and wound contraction, and increases collagen secretion [23] associated with chronic inflammation from the anti-fibrotic process. In our study, we showed that the candidates treated with their autologous ADMSC embedded in PRF improved significantly with an efficient wound healing process (Figure 4) compared to their counterparts treated only with PRF (Figure 3). Interestingly, this is the first clinical study in Lebanon to explore the synergic role of MSCs with Vivostat ${ }^{\circledR}$ PRF product on DFU healing.

Within our study, all DFU candidates received PRF injections, whether alone or with ADMSC, using the Vivostat ${ }^{\circledR}$ PRF system. The process for retrieving the fibrin from Vivostat ${ }^{\circledR}$ PRF provides a number of advantages compared with other automatic and manual systems used for obtaining PRF [24] [25]. First, the standardization of the PRP collection on Vivostat ${ }^{\circledR}$ PRF automated system provides a final product (PRP + autologous fibrin) stable in constitution indepen- 
dently of collection time delays and variations in handling operators. Second, the closed system feature in Vivostat ${ }^{\circledR}$ PRF provides a sterile therapeutic product, which minimizes the risk of possible microbial infection after PRF application. Remarkably, the concentration of both PRP and fibrin recovered after a Vivostat run is nine times fold the plasmatic concentration threshold, which is a considerably high value compared to other automatic PRF collection systems [22].

Eppley et al. performed a meta-analysis on three studies in which chronic ulcers were treated with PRF for 8 to 10 weeks and recorded complete epithelization of wounds in $81 \%, 93 \%$ and $100 \%$ of patients, respectively [26]. Similarly, our results showed good recovering scores after the completion of the PRF applications in four of the five candidates treated with PRF alone (Group A). One patient showed a low healing score, which may be correlated to the derived complication of his essential thrombocythemia. The mean concentration of TGF- $\beta 1$, PDGF-AB, PDGF-BB, VEGF and FGF- 2 secreted were statistically significant and correlated positively with proper DFU wound healing. The anti-bacterial properties attributed to PRF in other studies cannot be completely confirmed in our study, since only one candidate out of 10 showed a positive microbiological infection with Proteus mirabilis. Interestingly, the antibiotic therapy against $P$. mirabilis infection was not a conclusive factor in obtaining a poor score of healing recovery.

The five patients of Group B were injected with ADMSC embedded in PRF. Four candidates over five showed excellent wound healing scores (80\%) and only one candidate showed a good wound healing score (20\%) (Figure 4). It has been demonstrated that MSC-based treatments in wound healing reduced scarring, which may have accelerated the wound recovery and improved angiogenesis, modified collagen deposition, and paracrine signaling of MSCs [27]. Our results are consistent with many reports suggesting that MSCs secrete high levels of VEGF and HGF and maintain a higher ratio of TGF- $\beta 3$ to TGF- $\beta 1$, which contributes to cell migration, proliferation, and regulates vascular angiogenesis, matrix deposition, and degradation of wound healing; they also promote wound reepithelization and reduce scar formation [11]. FGF and HGF enhance the regeneration of the dermis in acute incisional wounds [28]. Thus, an increased VEGF is associated with scarless repair [29], as in neutralization of TGF- $\beta 1$ and TGF- $\beta 2$ or an increase of TGF- $\beta 3$ secretion. MSCs and PRF showed a high secretion of TGF- $\beta 1$, PDGF-AB, PDGF-BB, VEGF, and FGF-2 (Figure 1) promoting a fast wound healing in both groups. This is consistent with the latest theory stating that the therapeutic effects of MSCs are due to their paracrine effects and not due to their differentiation capacity. In fact, in the 2019 Caplan report [30], it was suggested to change the name of stem cells to medicinal signaling cells. The array of secretion or secretome has multiple therapeutic effects such as anti-inflammatory, anti-proliferative and anti-fibrotic effects besides the many other roles such as promoting anti scaring

Finally, our results suggest that in both groups, A and B, PRF and ADMSC 
with PRF promoted wound healing in DFUs. We should highlight the fact that the promising results obtained in our study that platelet-derived growth factors can replace conventional treatments (antibiotic creams, and skin or flap grafts) as a front-line therapeutic option in the treatment of chronic DFUs should not necessarily be generalized. PRF associated with MSC factors are a hopeful treatment protocol when conventional treatments are unsuccessful or when the patient refuses surgical options.

\section{Conclusions}

In conclusion, mesenchymal stem cells derived from adipose tissue and their secretions embedded in PRF potentially appear to have a major role in wound healing in DFU. The Vivostat ${ }^{\circledR}$ PRF system was used as a clinical and therapeutic tool for wound healing applications in our patients. In the future, it would be very interesting to conduct a prospective study in a larger number of patients, to statistically validate the use of ADMSCs embedded in PRF for the treatment of chronic DFU. Further studies are also needed to elucidate the underlying mechanism of cross-talkingbetween MSC secretome components and platelets growth factors.

Although many controversies have been reported on the role of stem cells in regenerative medicine, it is crucial that researchers continue to examine the roles and mechanisms of MSCs in wound healing to evaluate the therapeutic potential of MSCs and PRF.

\section{Conflicts of Interest}

The authors declare no conflicts of interest regarding the publication of this paper.

\section{References}

[1] Lipsky, B.A., Berendt, A.R., Cornia, P.B., Pile, J.C., Peters, E.J.G., Armstrong, D.G., et al. (2012) Infectious Diseases Society of America Clinical Practice Guideline for the Diagnosis and Treatment of Diabetic Foot Infections. Clinical Infectious Diseases, 54, e132-e173. https://doi.org/10.1093/cid/cis346

[2] Çamur, S., Batlbay, S.G. and Bayram, S. (2020) Effect of Lower Extremity Amputation on Caregiving Burden in Caregivers of Patients with Diabetic Foot: Prospective Cohort Study. International Wound Journal, 17, 890-896.

https://doi.org/10.1111/iwj.13342

[3] Hicks, C.W., Selvarajah, S., Mathioudakis, N., Sherman, R.L., Hines, K.F., Black, J.H., et al. (2016) Burden of Infected Diabetic Foot Ulcers on Hospital Admissions and Costs. Annals of Vascular Surgery, 33, 149-158.

https://doi.org/10.1016/j.avsg.2015.11.025

[4] Obeid, M., Moughames, E., Aboulhosn, P., Madi, R., Farah, M., Feghali, J., et al. (2018) Epidemiology and Susceptibility Profiles of Diabetic Foot Infections in Five Hospitals in Lebanon. The Journal of Infection in Developing Countries, 12, 347-351. https://doi.org/10.3855/jidc.10063

[5] Ubbink, D.T., Vermeulen, H. and Lubbers, M.J. (2006) Local Wound Care: Evi- 
dence-Based Treatments and Dressings. Nederlands Tijdschrift voor Geneeskunde, 150, 1165-1172.

[6] Haleem, A.M., Singergy, A.E., Sabry, D., Atta, H.M., Rashed, L.A., Chu, C.R., et al. (2010) The Clinical Use of Human Culture-Expanded Autologous Bone Marrow Mesenchymal Stem Cells Transplanted on Platelet-Rich Fibrin Glue in the Treatment of Articular Cartilage Defects: A Pilot Study and Preliminary Results. Cartilage, 1, 253-261. https://doi.org/10.1177/1947603510366027

[7] Srinivas, B., Das, P., Rana, M.M., Qureshi, A.Q., Vaidya, K.C. and Ahmed Raziuddin, S.J. (2018) Wound Healing and Bone Regeneration in Postextraction Sockets with and without Platelet-Rich Fibrin. Annals of Maxillofacial Surgery, 8, 28-34. https://doi.org/10.4103/ams.ams 15317

[8] Zhao, J.-H., Tsai, C.-H. and Chang, Y.-C. (2011) Clinical and Histologic Evaluations of Healing in an Extraction Socket Filled with Platelet-Rich Fibrin. Journal of Dental Sciences, 6, 116-122. https://doi.org/10.1016/j.jds.2011.03.004

[9] Wallace, H.A., Basehore, B.M. and Zito, P.M. (2020) Wound Healing Phases. StatPearls Publishing, Treasure Island. http://www.ncbi.nlm.nih.gov/books/NBK470443

[10] Gonzalez, A.C.O., Costa, T.F., Andrade, Z.A. and Medrado, A.R.A.P. (2016) Wound Healing-A Literature Review. Anais Brasileiros de Dermatologia, 91, 614-620. https://doi.org/10.1590/abd1806-4841.20164741

[11] Full Article: Role of Growth Factors and Biomaterials in Wound Healing.

[12] Follo, F., et al. (2017) Management and Effect of Platelet-Rich Plasma on Wound Healing: Small Reality of Oglio Po Hospital. Acta Bio Medica, 88, 66-70.

[13] Gao, F., Chiu, S.M., Motan, A.L., Zhang, Z., Chen, L., Ji, H.-L., et al. (2016) Mesenchymal Stem Cells and Immunomodulation: Current Status and Future Prospects. Cell Death \& Disease, 7, e2062. http://www.nature.com/articles/cddis2015327 https://doi.org/10.1038/cddis.2015.327

[14] Amati, E., Sella, S., Perbellini, O., Alghisi, A., Bernardi, M., Chieregato, K., et al. (2017) Generation of Mesenchymal Stromal Cells from Cord Blood: Evaluation of in Vitro Quality Parameters Prior to Clinical Use. Stem Cell Research \& Therapy, 8, 14. https://www.ncbi.nlm.nih.gov/pmc/articles/PMC5260040 https://doi.org/10.1186/s13287-016-0465-2

[15] Grigore, T.V. and Cozma, C. (2018) Platelet-Rich Plasma as a Site-Targeted Approach in Wound Healing: A Molecular Perspective. Discoveries (Craiova), 6, e87. https://doi.org/10.15190/d.2018.8

[16] Wang, X., Zhang, Y., Choukroun, J., Ghanaati, S. and Miron, R.J. (2018) Effects of an Injectable Platelet-Rich Fibrin on Osteoblast Behavior and Bone Tissue Formation in Comparison to Platelet-Rich Plasma. Platelets, 29, 48-55. https://doi.org/10.1080/09537104.2017.1293807

[17] Leach, J.K. and Whitehead, J. (2017) Materials-Directed Differentiation of Mesenchymal Stem Cells for Tissue Engineering and Regeneration. ACS Biomaterials Science \& Engineering, 4, 1115-1127.

[18] Fuertes, M., Velasco, V., Larrañaga, G.-E. and Peirona, G. (2009) Our Experience in the Treatment of Chronic Ulcers Using Vivostat ${ }^{\circledR} \mathrm{PRF}^{\circledR}$. Series of 10 Cases. Cirugía Plástica Ibero-Latinoamericana, 35, 9.

[19] Basic Principles of Wound Management-UpToDate. https://www.uptodate.com/contents/basic-principles-of-wound-management

[20] Etulain, J. (2018) Platelets in Wound Healing and Regenerative Medicine. Platelets, 29, 556-568. https://doi.org/10.1080/09537104.2018.1430357 
[21] Mizukami, H. and Yagihashi, S. (2014) Exploring a New Therapy for Diabetic Polyneuropathy-The Application of Stem Cell Transplantation. Frontiers in Endocrinology, 5, 45. https://www.ncbi.nlm.nih.gov/pmc/articles/PMC3988365 https://doi.org/10.3389/fendo.2014.00045

[22] Evaluation of the Effect of Vivostat Platelet Rich Fibrin (PRF) in the Treatment of Diabetic Foot Ulcers-Full Text View-ClinicalTrials.gov.

https://clinicaltrials.gov/ct2/show/NCT00770939

[23] Hu, M.S., Borrelli, M.R., Lorenz, H., Longaker, M.T. and Wan, D.C. (2018) Mesenchymal Stromal Cells and Cutaneous Wound Healing: A Comprehensive Review of the Background, Role, and Therapeutic Potential. Stem Cells International, 2018, Article ID: 6901983. https://www.hindawi.com/journals/sci/2018/6901983 https://doi.org/10.1155/2018/6901983

[24] Leitner, G.C., Gruber, R., Neumüller, J., Wagner, A., Kloimstein, P., Höcker, P., et al. (2006) Platelet Content and Growth Factor Release in Platelet-Rich Plasma: A Comparison of Four Different Systems. Vox Sanguinis, 91, 135-139. https://doi.org/10.1111/j.1423-0410.2006.00815.x

[25] Zimmermann, R., Jakubietz, R., Jakubietz, M., Strasser, E., Schlegel, A., Wiltfang, J., et al. (2001) Different Preparation Methods to Obtain Platelet Components as a Source of Growth Factors for Local Application. Transfusion, 41, 1217-1224. https://doi.org/10.1046/j.1537-2995.2001.41101217.x

[26] Eppley, B.L., Woodell, J.E. and Higgins, J. (2004) Platelet Quantification and Growth Factor Analysis from Platelet-Rich Plasma: Implications for Wound Healing. Plastic and Reconstructive Surgery, 114, 1502-1508. https://doi.org/10.1097/01.PRS.0000138251.07040.51

[27] Du, H.-C., et al. (2017) Growth Factor-Reinforced ECM Fabricated from Chemically Hypoxic MSC Sheet with Improved in Vivo Wound Repair Activity. BioMed Research International, 2017, Article ID: 2578017. https://www.hindawi.com/journals/bmri/2017/2578017

[28] Ono, I., Yamashita, T., Hida, T., Jin, H.-Y., Ito, Y., Hamada, H., et al. (2004) Combined Administration of Basic Fibroblast Growth Factor Protein and the Hepatocyte Growth Factor Gene Enhances the Regeneration of Dermis in Acute Incisional Wounds. Wound Repair and Regeneration, 12, 67-79. https://doi.org/10.1111/j.1067-1927.2004.012113.x-1

[29] Rolfe, K.J. and Grobbelaar, A.O. (2012) A Review of Fetal Scarless Healing. International Scholarly Research Notices, 2012, Article ID: 698034.

https://www.hindawi.com/journals/isrn/2012/698034 https://doi.org/10.5402/2012/698034

[30] Caplan, A.I. (2019) Medicinal Signalling Cells: They Work, So Use Them. Nature, 566, 39. https://doi.org/10.1038/d41586-019-00490-6 